TI 2011-052/ 1

Tinbergen Institute Discussion Paper

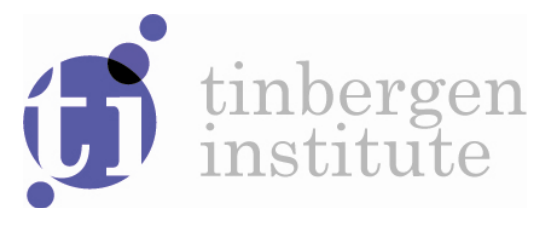

\title{
An Efficient and Fair Solution for Communication Graph Games
}

René van den Brink ${ }^{7}$

Anna Khmelnitskaya ${ }^{2}$

Gerard van der Laan'

' VU University Amsterdam, and Tinbergen Institute, the Netherlands;

2 Russian Academy of Sciences, St. Petersburg, Russia. 
Tinbergen Institute is the graduate school and research institute in economics of Erasmus University Rotterdam, the University of Amsterdam and VU University Amsterdam.

More TI discussion papers can be downloaded at http://www.tinbergen.nl

Tinbergen Institute has two locations:

Tinbergen Institute Amsterdam

Gustav Mahlerplein 117

1082 MS Amsterdam

The Netherlands

Tel.: +31(0)205251600

Tinbergen Institute Rotterdam

Burg. Oudlaan 50

3062 PA Rotterdam

The Netherlands

Tel.: +31(0)10 4088900

Fax: +31(0)104089031

Duisenberg school of finance is a collaboration of the Dutch financial sector and universities, with the ambition to support innovative research and offer top quality academic education in core areas of finance.

DSF research papers can be downloaded at: http://www.dsf.nl/

Duisenberg school of finance

Gustav Mahlerplein 117

1082 MS Amsterdam

The Netherlands

Tel.: +31(0)20 5258579 


\title{
An efficient and fair solution for communication graph games $^{1}$
}

\author{
René van den Brink ${ }^{2}$ Anna Khmelnitskaya ${ }^{3}$ Gerard van der Laan ${ }^{4}$
}

March 11, 2011

\footnotetext{
${ }^{1}$ This research was supported by NWO (The Netherlands Organization for Scientific Research) grant NL-RF 047.017.017. The research of Anna Khmelnitskaya was also partially supported by CRT Foundation and the University of Eastern Piedmont at Alessandria through the Lagrange Project 2010.

${ }^{2}$ J.R. van den Brink, Department of Econometrics and Tinbergen Institute, VU University, De Boelelaan 1105, 1081 HV Amsterdam, The Netherlands. E-mail: jrbrink@feweb.vu.nl

${ }^{3}$ A.B. Khmelnitskaya, SPb Institute for Economics and Mathematics Russian Academy of Sciences, Tchaikovsky st. 1, 191187 St.Petersburg, Russia, E-mail: a.khmelnitskaya@math.utwente.nl

${ }^{4}$ G. van der Laan, Department of Econometrics and Tinbergen Institute, VU University, De Boelelaan 1105, 1081 HV Amsterdam, The Netherlands. E-mail: glaan@feweb.vu.nl
} 


\begin{abstract}
We introduce an efficient solution for games with communication graph structures and show that it is characterized by efficiency, fairness and a new axiom called component balancedness. This latter axiom compares for every component in the communication graph the total payoff to the players of this component in the game itself to the total payoff of these players when applying the solution to the subgame induced by this component.
\end{abstract}

Keywords: TU game, communication graph, Myerson value, fairness, efficiency.

JEL code: $\mathrm{C} 71$ 


\section{Introduction}

A situation in which a finite set of players can obtain certain payoffs by cooperation can be described by a cooperative game with transferable utility, or simply a TU-game, being a pair consisting of a finite set of players and a characteristic function on the collection of all coalitions of players, that assigns a worth to each coalition of players. In this note we consider TU-games with limited cooperation possibilities, represented by an undirected communication graph, as introduced by Myerson [7]. The nodes in the graph represent the players and the edges represent the communication links between the players. Players can only cooperate if they are connected. This yields a so-called (communication) graph game, given by a triple consisting of a finite set of players, a characteristic function and a communication graph.

A (single-valued) solution for communication graph games is a mapping that assigns to every communication graph game a payoff vector. The best-known solution for commu-

nication graph games is the Myerson value [7], which is obtained as the Shapley value of a restricted game, and is characterized by component efficiency and fairness. Component efficiency states that for each component of the communication graph the total payoff to the players of the component is equal to the worth of that component in the characteristic function. Fairness says that deleting a link between two players yields for both players the same change in payoff. Another single-valued solution concept, the so-called position value, is introduced in Meessen [6] and developed in Borm, Owen and Tijs [1]. Slikker [10] axiomatizes the position value using component efficiency and balanced total threats. For cycle-free communication graph games, Herings, van der Laan and Talman [4] introduced the so-called Average Tree solution, characterized by component efficiency and component fairness, the latter axiom stating that deleting a link between two players in a cycle-free graph game yields the same average change in payoff in the two components that result from deleting the link. All these solutions satisfy component efficiency. Therefore, efficiency is only guaranteed when the graph is connected and thus contains the player set itself as its unique component.

In contrast to the reasoning that a set of players can only realise its worth when they are connected, and thus eventually the players in each component distribute the worth of that component among each other, in some situations efficiency is obtained, even when the communication graph is not connected. As an example, consider a research fund that has an amount of money available to distribute amongst individual researchers. Every researcher that submits a proposal takes part in the distribution of the available budget, so writing an individual proposal is the only requirement for a researcher to get access to the fund. However, the board of the research fund has the policy to stimulate interdisciplinary research and therefore joint proposals get priority. Researchers can secure some part of the 
fund by submitting joint proposals. For instance, suppose that the budget of the fund is 12 and there are three researchers, named A, B and C. An individual proposal just gives access to the fund, but does not secure any amount of money. On the other hand, A en $\mathrm{B}$ can secure themselves a grant of 3 when writing a joint proposal, $\mathrm{A}$ and $\mathrm{C}$ a grant of 2 and $\mathrm{B}$ and $\mathrm{C}$ a grant of 4 . However, $\mathrm{C}$ does not communicate with the others, so the only feasible coalition is $\mathrm{A}$ and $\mathrm{B}$ and the communication graph consists of two components: the coalition of $\mathrm{A}$ and $\mathrm{B}$ that can secure themselves 3 , and the singleton agent $\mathrm{C}$ that can only secure itself 0 . According to the Myerson value the total amount of money granted to the researchers is only 3 , but in this situation the board of the research fund will grant 3 to $\mathrm{A}$ and $\mathrm{B}$ and will then distribute the remaining 9 to the researchers. Although the communication graph is not connected, the full budget of 12 is still available to the coalition of all players. So, this requires a value satisfying efficiency.

Recently, also Casajus [2] argued by some motivating example that in some situations it seems reasonable to require efficiency, even when the communication graph is not connected and thus has multiple components. He introduced a solution for communication graph games that is characterized by efficiency, equivalence (meaning that the total payoff in case of the complete graph is equal to the total payoff in case of the empty graph), component merging (meaning that merging the components' players into a single player does not affect the total payoff to the component) and a modified version of Myerson's fairness. In this note we introduce a new solution for communication graph games that, besides efficiency and Myerson's fairness, satisfies a new axiom called component balancedness. This component balancedness axiom compares for every component in the communication graph the total payoff to the players of this component in the game itself to the total payoff of this component when applying the solution to the subgame induced by this component. It also can be seen as weak version of component efficiency. The new solution equals the Shapley value when the graph is connected and is equal to the equal surplus division when the graph is empty.

This note is organized as follows. Basic definitions and notation are introduced in Section 2. The component balancedness axiom, the new solution and its characterization are given in Section 3. At the end of that section we return to the research fund example described above and compare our solution with several others.

\section{Preliminaries}

A situation in which a finite set of players can obtain certain payoffs by cooperating can be described by a cooperative game with transferable utility, or simply a TU-game, being a pair $\langle N, v\rangle$, where $N \subset \mathbb{N}$ is a finite set of $n \geq 2$ players and $v: 2^{N} \rightarrow \mathbb{R}$ is a characteristic 
function on $N$ such that $v(\emptyset)=0$. For any coalition $S \subseteq N, v(S)$ is the worth of coalition $S$, i.e., the members of coalition $S$ can obtain a total payoff of $v(S)$ by agreeing to cooperate.

We denote the set of all characteristic functions on given player set $N$ by $\mathcal{G}^{N}$. Although $N$ is not fixed, nevertheless for simplicity of notation and if no ambiguity appears, we write $v$ instead of $\langle N, v\rangle$. For given $N$, the subgame of a game $v \in \mathcal{G}^{N}$ with respect to a player set $T \subset N, T \neq \emptyset$, is the game $v_{T} \in \mathcal{G}^{T}$ defined as $v_{T}(S)=v(S)$, for all $S \subseteq T$. We denote the cardinality of a given set $A$ by $|A|$, along with lower case letters like $n=|N|$, $c=|C|, c^{\prime}=\left|C^{\prime}\right|$ and so on. For $K \subset \mathbb{N}$, we denote $\mathbb{R}^{\mathrm{K}}$ as the $k$-dimensional vector space which elements $x \in \mathbb{R}^{\mathrm{K}}$ have components $x_{i}, i \in K$.

For game $v \in \mathcal{G}^{N}$, a vector $x \in \mathbb{R}^{N}$ may be considered as a payoff vector assigning a payoff $x_{i}$ to each player $i \in N$. A single-valued solution, called a value, is a mapping $\xi$ that assigns for every $N$ and every $v \in \mathcal{G}^{N}$ a payoff vector $\xi(v) \in \mathbb{R}^{N}$. A value $\xi$ is efficient if $\sum_{i \in N} \xi_{i}(v)=v(N)$ for every $v \in \mathcal{G}^{N}$ and $N \subset \mathbb{N}$. The best-known efficient value is the Shapley value [9], given by

$$
S h_{i}(v)=\sum_{\{S \subseteq N \mid i \in S\}} \frac{(n-s) !(s-1) !}{n !}(v(S)-v(S \backslash\{i\})), \quad \text { for all } i \in N .
$$

For $N \subset \mathbb{N}$, a communication structure on $N$ is specified by a communication graph $\langle N, \Gamma\rangle$ with $\Gamma \subseteq \Gamma^{N}=\{\{i, j\} \mid i, j \in N, i \neq j\}$, i.e., $\Gamma$ is a collection of (unordered) pairs of nodes (players), where a pair $\{i, j\}$ represents a link between players $i, j \in N$, and $\left\langle N, \Gamma^{N}\right\rangle$ is the complete graph on $N$. Again, for simplicity of notation and if no ambiguity appears, we write graph $\Gamma$ instead of $\langle N, \Gamma\rangle$. Let $\mathcal{L}^{N}$ denote the set of all communication graphs on $N$. A pair $\langle v, \Gamma\rangle \in \mathcal{G}^{N} \times \mathcal{L}^{N}$ constitutes a game with (communication) graph structure or simply a graph game on $N$. For given $N$, the subgraph of a graph $\Gamma \in \mathcal{L}^{N}$ with respect to set $T \subseteq N, T \neq \emptyset$, is the graph $\left.\Gamma\right|_{T} \in \mathcal{L}^{T}$ defined by $\left.\Gamma\right|_{T}=\{\{i, j\} \in \Gamma \mid i, j \in T\}$.

For a graph $\Gamma$, a sequence of different nodes $\left(i_{1}, \ldots, i_{k}\right), k \geq 2$, is a path from $i_{1}$ to $i_{k}$, if for all $h=1, \ldots, k-1,\left\{i_{h}, i_{h+1}\right\} \in \Gamma$. A graph $\Gamma$ on a player set $N$ is connected, if for any two nodes in $N$ there exists a path in $\Gamma$ from one node to the other. For given graph $\Gamma$ on $N$, we say that the player set $S \subseteq N$ is connected, if the subgraph $\left.\Gamma\right|_{S}$ is connected. For graph $\Gamma$ on player set $N$ and $S \subseteq N$, a subset $T \subseteq S$ is a component of $S$ if (i) $\left.\Gamma\right|_{T}$ is connected, and (ii) for every $i \in S \backslash T$, the subgraph $\left.\Gamma\right|_{T \cup\{i\}}$ is not connected. For $\Gamma$ on $N$ and $S \subseteq N$, we denote by $S / \Gamma$ the set of all components of $S$, and by $(S / \Gamma)_{i}$ the component of $S$ containing $i \in S$. Notice that $S / \Gamma$ is a partition of $S$.

A single-valued solution for communication graph games, a graph game value, is a mapping $\xi$ that for every $N \subset \mathbb{N}$ and every $\langle v, \Gamma\rangle \in \mathcal{G}^{N} \times \mathcal{L}^{N}$ assigns a payoff vector $\xi(v, \Gamma) \in \mathbb{R}^{N}$. A well-known graph game value is the Myerson value. In Myerson [7] it is assumed that in a communication graph game $\langle v, \Gamma\rangle$ only connected coalitions are able to cooperate and to realise their worths. A non-connected coalition $S$ can only realise the sum 
of the worths of its components in $S / \Gamma$. This yields the restricted game $v^{\Gamma} \in \mathcal{G}^{N}$ defined by

$$
v^{\Gamma}(S)=\sum_{T \in S / \Gamma} v(T), \quad \text { for all } S \subseteq N .
$$

Then the Myerson value for communication graph games is the graph game value $\mu$ that assigns to every communication graph game $\langle v, \Gamma\rangle$ the Shapley value of its restricted game $v^{\Gamma}$, i.e.,

$$
\mu(v, \Gamma)=S h_{i}\left(v^{\Gamma}\right) \quad \text { for all }\langle v, \Gamma\rangle \in \mathcal{G}^{N} \times \mathcal{L}^{N} \text { and every } N \subset \mathbb{N} .
$$

It is well-known that the Myerson value is the unique graph game value that is component efficient and satisfies the so-called Myerson fairness axiom. The aim of this paper is to introduce an efficient and fair solution for communication graph games.

To conclude the introduction section we recall definitions of efficiency, component efficiency and fairness. A graph game value $\xi$ is

- efficient if for every graph game $\langle v, \Gamma\rangle$ on any player set $N, \sum_{i \in N} \xi_{i}(v, \Gamma)=v(N)$;

- component efficient if for every graph game $\langle v, \Gamma\rangle$ on any player set $N$, for every $C \in N / \Gamma, \sum_{i \in C} \xi_{i}(v, \Gamma)=v(C)$;

- fair if for every graph game $\langle v, \Gamma\rangle$ on any player set $N$, for every $\{h, k\} \in \Gamma$, $\xi_{h}(v, \Gamma)-\xi_{h}\left(v, \Gamma_{-h k}\right)=\xi_{k}(v, \Gamma)-\xi_{k}\left(v, \Gamma_{-h k}\right)$, where $\Gamma_{-h k}=\Gamma \backslash\{\{h, k\}\}$.

\section{Efficiency, fairness and component balancedness}

In this note we look for a graph game value that is characterized by efficiency, fairness and a new axiom that we refer to as component balancedness.

Component balancedness (CB) For every graph game $\langle v, \Gamma\rangle$ on any player set $N$, for every component $C \in N / \Gamma$, it holds

$$
\frac{\sum_{i \in C}\left(\xi_{i}(v, \Gamma)-\xi_{i}\left(v_{C},\left.\Gamma\right|_{C}\right)\right)}{c}=\frac{\sum_{i \in N}\left(\xi_{i}(v, \Gamma)-\xi_{i}\left(v_{(N / \Gamma)_{i}},\left.\Gamma\right|_{(N / \Gamma)_{i}}\right)\right.}{n} .
$$

First, note that this axiom only states a requirement on the payoffs when the collection of components $N / \Gamma$ contains at least two elements, otherwise the requirement reduces to an identity. Further, notice that the games $\left\langle v_{C},\left.\Gamma\right|_{C}\right\rangle$ and $\left\langle v_{(N / \Gamma)_{i}},\left.\Gamma\right|_{(N / \Gamma)_{i}}\right\rangle$ are defined on the reduced player sets $C$, respectively, $(N / \Gamma)_{i}$. For a component $C \in N / \Gamma$, the axiom compares the payoffs that the players of $C$ receive in the game itself to the payoffs that 
these players receive in the subgame on $C$. Considering two components $C, C^{\prime} \in N / \Gamma$, this axiom implies that

$$
\frac{1}{c} \sum_{i \in C}\left(\xi_{i}(v, \Gamma)-\xi_{i}\left(v_{C},\left.\Gamma\right|_{C}\right)\right)=\frac{1}{c^{\prime}} \sum_{i \in C^{\prime}}\left(\xi_{i}(v, \Gamma)-\xi_{i}\left(v_{C^{\prime}},\left.\Gamma\right|_{C^{\prime}}\right)\right),
$$

meaning that considering only the players in component $C$, the change in the average payoff of the players in this component is the same as the change in the average payoff of the players in any other component $C^{\prime}$ resulting from considering only the players in that component $C^{\prime}$. We refer to this axiom as component balancedness because it has some flavour of the balanced contributions ${ }^{1}$ property of Myerson [8], but in terms of the average change of payoffs in components. ${ }^{2}$ Component balancedness also can be seen as a weak version of component efficiency since every graph game value that satisfies component efficiency satisfies component balancedness. This follows straightforward since component efficiency implies that $\sum_{i \in C} \xi_{i}(v, \Gamma)=\sum_{i \in C} \xi_{i}\left(v_{C},\left.\Gamma\right|_{C}\right)=v(C)$, for all $C \in N / \Gamma$.

As mentioned, we will show that there is a unique graph game value that satisfies efficiency, fairness and component balancedness. This solution is obtained by taking the Shapley value of a slight modification of the restricted game $v^{\Gamma}$. If we want to obtain an efficient graph game value as the Shapley value of some restricted game, then at least the worth of the 'grand coalition' $N$ in the restricted game must be $v(N)$. It turns out that this modification is sufficient to obtain the unique graph game value satisfying efficiency, fairness and component balancedness. So, for a player set $N \subset \mathbb{N}$ and $\langle v, \Gamma\rangle \in \mathcal{G}^{N} \times \mathcal{L}^{N}$, we define $\bar{v}^{\Gamma} \in \mathcal{G}^{N}$ by $^{3}$

$$
\bar{v}^{\Gamma}(S)= \begin{cases}v^{\Gamma}(S), & S \varsubsetneqq N, \\ v(N), & S=N,\end{cases}
$$

and consider the graph game value $\psi$ given by

$$
\psi(v, \Gamma)=S h_{i}\left(\bar{v}^{\Gamma}\right) \quad \text { for all }\langle v, \Gamma\rangle \in \mathcal{G}^{N} \times \mathcal{L}^{N} \text { and every } N \subset \mathbb{N} \text {. }
$$

We then have the following theorem.

\footnotetext{
${ }^{1}$ Balanced contributions for communication graph games states that isolating a player, say $i$, in the communication structure has the same effect on the payoffs of another player, say $j$, as the effect on the payoff of $i$ as a result of isolating player $j$, i.e., for every graph game $\langle v, \Gamma\rangle$ and $i, j \in N$, it holds that $\xi_{i}(v, \Gamma)-\xi_{i}\left(v, \Gamma \backslash \Gamma_{j}\right)=\xi_{j}(v, \Gamma)-\xi_{j}\left(v, \Gamma \backslash \Gamma_{i}\right)$, where $\Gamma_{h}=\{\{i, j\} \in \Gamma \mid h \in\{i, j\}\}$.

${ }^{2}$ It is worth remarking that component balancedness also has the flavor of several other known types of axioms such as consistency (looking at reduced games, but not saying that the payoffs of remaining players do not change) or component fairness (comparing average changes of payoffs in a component, but not after link deletion).

${ }^{3}$ So, $\bar{v}^{\Gamma}$ is the Myerson restricted game, except that $\bar{v}^{\Gamma}(N)=v(N)$ instead of $\sum_{H \in N / \Gamma} v(H)$.
} 
Theorem 3.1 The graph game value $\psi$ is efficient, fair and satisfies component balancedness.

Proof. Since $\bar{v}^{\Gamma}(N)=v(N)$, efficiency follows by efficiency of the Shapley value. So, we only have to show fairness and component balancedness. By definition we have that $\bar{v}^{\Gamma}=v^{\Gamma}+w$, where $w \in \mathcal{G}^{N}$ is given by

$$
w(S)= \begin{cases}0, & S \varsubsetneqq N, \\ v(S)-v^{\Gamma}(S), & S=N,\end{cases}
$$

i.e., game $\bar{v}^{\Gamma}$ is obtained by adding $v(N)-v^{\Gamma}(N)$ times the unanimity game $\mathrm{e}^{4}$ of $N$ to the Myerson restricted game $v^{\Gamma}$. From this and the additivity and symmetry properties of the Shapley value it follows that

$$
\psi_{i}(v, \Gamma)=S h_{i}\left(\bar{v}^{\Gamma}\right)=S h_{i}\left(v^{\Gamma}\right)+S h_{i}(w)=\mu_{i}(v, \Gamma)+\frac{v(N)-v^{\Gamma}(N)}{n},
$$

where the last equality follows by definition of $\mu$ and $w$. Hence,

$$
\begin{aligned}
\psi_{i}(v, \Gamma)-\psi_{i}\left(v, \Gamma_{-i j}\right) & =\mu_{i}(v, \Gamma)+\frac{v(N)-v^{\Gamma}(N)}{n}-\left(\mu_{i}\left(v, \Gamma_{-i j}\right)+\frac{v(N)-v^{\Gamma_{-i j}}(N)}{n}\right) \\
& =\mu_{i}(v, \Gamma)-\mu_{i}\left(v, \Gamma_{-i j}\right)-\frac{v^{\Gamma}(N)-v^{\Gamma_{-i j}}(N)}{n} \\
& =\mu_{j}(v, \Gamma)-\mu_{j}\left(v, \Gamma_{-i j}\right)-\frac{v^{\Gamma}(N)-v^{\Gamma_{-i j}}}{n}=\psi_{j}(v, \Gamma)-\psi_{j}\left(v, \Gamma_{-i j}\right),
\end{aligned}
$$

where the third equality follows by fairness of $\mu$. Hence, $\psi$ satisfies fairness.

To show component balancedness, by (3.1) we obtain for every $C \in N / \Gamma$ that

$$
\sum_{i \in C} \psi_{i}(v, \Gamma)=\sum_{i \in C} \mu_{i}(v, \Gamma)+\frac{c}{n}\left(v(N)-v^{\Gamma}(N)\right) .
$$

Further, $\sum_{i \in C} \mu_{i}(v, \Gamma)=v(C)$ because of component efficiency of the Myerson value, and the total payoff that $\psi$ assigns to the players in $C$ in the subgame $\left\langle v_{C},\left.\Gamma\right|_{C}\right\rangle$ is equal to $v(C)$ because of the efficiency of $\psi$ itself. Thus, with (3.1)

$$
\sum_{i \in C}\left(\psi_{i}(v, \Gamma)-\psi_{i}\left(v_{C},\left.\Gamma\right|_{C}\right)\right)=v(C)+\frac{c}{n}\left(v(N)-v^{\Gamma}(N)\right)-v(C)=\frac{c}{n}\left(v(N)-v^{\Gamma}(N)\right) .
$$

Also, by efficiency of $\psi$ we have $\sum_{i \in N} \psi_{i}\left(v_{(N / \Gamma)}, \Gamma_{(N / \Gamma)_{i}}\right)=\sum_{H \in N / \Gamma} \sum_{i \in H} \psi_{i}\left(v_{H},\left.\Gamma\right|_{H}\right)=$ $\sum_{H \in N / \Gamma} v(H)$, and thus

$$
\sum_{i \in N}\left(\psi_{i}(v, \Gamma)-\psi_{i}\left(v_{(N / \Gamma)_{i}}, \Gamma_{(N / \Gamma)_{i}}\right)\right)=v(N)-\sum_{H \in N / \Gamma} v(H)=v(N)-v^{\Gamma}(N) .
$$

\footnotetext{
${ }^{4}$ It is well known from [9] that the collection of unanimity games $\left\{u_{T}\right\}_{\substack{T \subset N \\ T \neq \emptyset}}$, defined as $u_{T}(S)=1$ if $T \subseteq S$, and $u_{T}(S)=0$ otherwise, form a basis in $\mathcal{G}^{N}$ in the sense that every $v \in \mathcal{G}^{N}$ can be written as a unique linear combination of unanimity games, where the coefficients are the Harsanyi dividends, see [3].
} 
Hence, $\psi$ satisfies component balancedness.

Note that (3.1) gives an alternative definition of the graph game value $\psi$ assigning to every graph game its Myerson value and distributing the difference between the worth of the 'grand coalition' $N$ and the sum of the worths of all components equally over all players. In this sense the solution $\psi$ can be seen as combining elements of the Shapley value and equal division solution. ${ }^{5}$ The next theorem characterizes the graph game value $\psi$.

Theorem 3.2 There is a unique graph game value $\xi$ satisfying efficiency, fairness and component balancedness.

Proof. By Theorem 3.1 we only need to show uniqueness. We first consider the case that $\Gamma$ is the empty graph. Then $N / \Gamma=\{\{i\} \mid i \in N\}$, i.e., every node is a singleton component, and component balancedness requires for every $i \in N$ that

$$
\xi_{i}(v, \Gamma)-\xi_{i}\left(v_{\{i\}},\left.\Gamma\right|_{\{i\}}\right)=\frac{1}{n} \sum_{j \in N}\left(\xi_{j}(v, \Gamma)-\xi_{j}\left(v_{\{j\}},\left.\Gamma\right|_{\{j\}}\right)\right) .
$$

By efficiency $\sum_{j \in N} \xi_{j}(v, \Gamma)=v(N)$ and also for every $j \in N, \xi_{j}\left(v_{\{j\}},\left.\Gamma\right|_{\{j\}}\right)=v(\{j\})$. Hence, when $\Gamma$ is the empty graph, then by (3.2) the payoffs

$$
\xi_{i}(v, \Gamma)=v(\{i\})+\frac{1}{n}\left(v(N)-\sum_{j \in N} v(\{j\})\right), \quad i \in N,
$$

are uniquely determined.

We now proceed by induction similar as in [7], but replacing component efficiency by efficiency and component balancedness. Consider graph game $\langle v, \Gamma\rangle \in \mathcal{G}^{N} \times \mathcal{L}^{N}$, and suppose that we determined the payoffs for every $\left\langle v^{\prime}, \Gamma^{\prime}\right\rangle \in \cup_{K \subseteq N} \mathcal{G}^{K} \times \mathcal{L}^{K}$ with $\left|\Gamma^{\prime}\right|<|\Gamma|$. Efficiency requires that

$$
\sum_{i \in N} \xi_{i}(v, \Gamma)=v(N)
$$

Further, for a component $C \in N / \Gamma$, component balancedness implies that

$$
\frac{\sum_{i \in C}\left(\xi_{i}(v, \Gamma)-\xi_{i}\left(v_{C},\left.\Gamma\right|_{C}\right)\right)}{c}=\frac{\sum_{i \in N}\left(\xi_{i}(v, \Gamma)-\xi_{i}\left(v_{(N / \Gamma)_{i}},\left.\Gamma\right|_{(N / \Gamma)_{i}}\right)\right.}{n} .
$$

\footnotetext{
${ }^{5}$ This idea is similar to Kamijo [5] who introduced a solution for games in coalition structure, i.e., the player set is partitioned into unions, that allocates to every player its Shapley value in the game restricted to its own union and distributes the Shapley value of its union in the (quotient) game between the unions equally among the players in each union. Considering the associated communication graph, being the graph where there is a link between any pair of players in the same union and no links between players in different unions, the unions are exactly the components in that communication graph.
} 
When $C=N$, this is an identity and the equation is redundant. Otherwise, efficiency requires that for every $H \in N / \Gamma$,

$$
\sum_{i \in H} \xi_{i}\left(v_{H},\left.\Gamma\right|_{H}\right)=v(H)
$$

Using the equations (3.4) and (3.6), equation (3.5) reduces to

$$
\sum_{i \in C} \xi_{i}(v, \Gamma)-v(C)=\frac{c}{n}\left(v(N)-\sum_{H \in N / \Gamma} v(H)\right) .
$$

Let $m=|N / \Gamma|$ be the number of components. Since summing up the equations (3.5) over all components yields an identity, the number of independent equations (3.7) is $m-1$. So, equations (3.4) and (3.7) yield together $m$ independent linear equations.

Next, let $\Gamma^{\prime}$ be a spanning subforest of $\Gamma$, i.e., $\Gamma^{\prime} \subseteq \Gamma$ with $\left|\Gamma^{\prime}\right|=n-m$, and $N / \Gamma^{\prime}=N / \Gamma$ (both forests have the same collection of components). Note that for every link $\{i, j\} \in \Gamma^{\prime}$ it holds that $\left|N / \Gamma_{-i j}^{\prime}\right|>\left|N / \Gamma^{\prime}\right|$ (deleting any link from $\Gamma^{\prime}$ increases the number of components). For every link $\{i, j\} \in \Gamma^{\prime}$, by fairness it holds that

$$
\xi_{i}(v, \Gamma)-\xi_{i}\left(v, \Gamma_{-i j}\right)=\xi_{j}(v, \Gamma)-\xi_{j}\left(v, \Gamma_{-i j}\right)
$$

Since $\left|\Gamma_{-i j}\right|=|\Gamma|-1$, for every $\{i, j\} \in \Gamma^{\prime}$ all values $\xi_{h}\left(v, \Gamma_{-i j}\right), h \in N$, have been determined by the induction hypothesis. Then (3.8) yields $n-m$ linearly independent equations. So, together the system of equations (3.4), (3.7) and (3.8) yield $1+(m-1)+$ $(n-m)=n$ linearly independent equations in $n$ unknown payoffs $\xi_{i}(v, \Gamma), i \in N$, and so all payoffs $\xi_{i}(v, \Gamma), i \in N$, are uniquely determined.

Notice from equation (3.3) that the solution $\xi$ divides the excess $v(N)-\sum_{j \in N} v(\{j\})$ equally among the players when the graph is empty, and thus yields the equal surplus division solution. On the other hand, when the graph is complete the solution $\xi$ gives the Shapley value of $v$.

For the example given in the introduction we have $v(\{i\})=0, i=A, B, C$, $v(\{A, B\})=3, v(\{A, C\})=2, v(\{B, C\})=4, v(N)=12$ and $\Gamma=\{\{A, B\}\}$. So $N / \Gamma=\{\{A, B\},\{C\}\}$. The Shapley value of $v$ is efficient and yields $S h(v)=\left(3 \frac{1}{2}, 4 \frac{1}{2}, 4\right)$, and the Myerson value is component efficient and yields $\mu(v, \Gamma)=\left(\frac{3}{2}, \frac{3}{2}, 0\right)$. The new solution is efficient and yields $\psi(v, \Gamma)=\left(4 \frac{1}{2}, 4 \frac{1}{2}, 3\right)$. Of course, efficiency requires that the total budget of 12 is allocated. Since all singleton worths are zero, fairness implies that the link between $\mathrm{A}$ and $\mathrm{B}$ gives them an equal payoff in this example ${ }^{6}$. So, we only need

\footnotetext{
${ }^{6}$ This follows since, as mentioned before, efficiency and component balancedness imply equal surplus division (every player gets its singleton worth and the remainder is equally distributed over all players) for the empty graph.
} 
to determine the shares in the total budget of $\mathrm{C}$ compared to $\mathrm{A}$ and $\mathrm{B}$ together. This is done by component balancedness which requires that the total payoff of $\mathrm{A}$ and $\mathrm{B}$ together minus their worth (being equal to 3 ) is twice the difference between the payoff of $\mathrm{C}$ and its worth, implying that $\mathrm{A}$ and $\mathrm{B}$ together get a fraction $\frac{2}{3}$ and $\mathrm{C}$ gets a fraction $\frac{1}{3}$ of the 'surplus' $12-3=9$. As can be seen in this example, our solution favors cooperation among players since the stand alone player $\mathrm{C}$ gets less than one third of the budget. The outcome of Casajus' value for this example is $\left(3 \frac{1}{4}, 4 \frac{1}{4}, 4 \frac{1}{2}\right)$, and thus the stand alone player $\mathrm{C}$ gets more than one third of the budget. This occurs because this solution favors (non cooperative) stand alone players.

Finally, we show logical independence of the axioms of Theorem 3.2. First, the Myerson value is fair and satisfies component balancedness (there is zero excess to divide among the components), but is not efficient. Second, the equal division solution given by $E D_{i}(v, \Gamma)=\frac{v(N)}{|N|}, i \in N,\langle v, \Gamma\rangle \in \mathcal{G}^{N} \times \mathcal{L}^{N}, N \subset \mathbb{N}$, is efficient and fair, but is not component balanced. Third, the component-wise equal division solution given by $C E D_{i}(v, \Gamma)=\frac{v\left((N / \Gamma)_{i}\right)}{\left|(N / \Gamma)_{i}\right|}+\frac{1}{n}\left(v(N)-\sum_{H \in N / \Gamma} v(H)\right), i \in N,\langle v, \Gamma\rangle \in \mathcal{G}^{N} \times \mathcal{L}^{N}, N \subset \mathbb{N}$, is efficient and component balanced, but not fair.

\section{References}

[1] Borm, P., Owen, G., Tijs, S. (1992), On the position value for communication situations, SIAM Journal on Discrete Mathemathics 5, 305-320.

[2] Casajus, A. (2007), An efficient value for TU games with a cooperation structure, Working paper, Universität Leipzig, Germany.

[3] Harsanyi, J.C. (1959), A bargaining model for cooperative $n$-person games, in: Tucker AW, Luce RD (eds.) Contributions to the theory of games IV, Princeton University Press, Princeton, NJ, pp. 325-355.

[4] Herings, P.J.J., van der Laan, G., Talman, A.J.J. (2008), The average tree solution for cycle-free graph games, Games and Economic Behavior 62, 77-92.

[5] Kamijo, Y. (2009), A two-step Shapley value for cooperative games with coalition structures, International Game Theory Review, 11, 207-214.

[6] Meessen, R. (1988), Communication games. Master Thesis, University of Nijmegen.

[7] Myerson, R.B. (1977), Graphs and cooperation in games, Mathematics of Operations Research 2, 225-229. 
[8] Myerson, R.B. (1980), Conference structures and fair allocation rules, International Journal of Game Theory 9, 169-182.

[9] Shapley, L.S. (1953), A value for $n$-person games, in: Tucker AW, Kuhn HW (eds.) Contributions to the theory of games II, Princeton University Press, Princeton, NJ, pp. 307-317.

[10] Slikker, M. (2005), A characterization of the position value, International Journal of Game Theory 33, 505-514. 